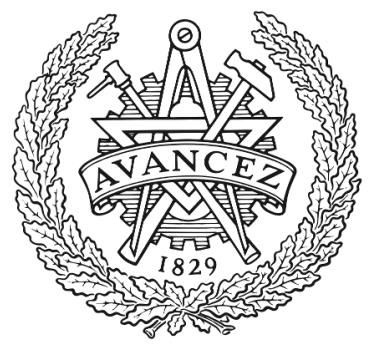

CHALMERS

UNIVERSITY OF TECHNOLOGY

\title{
Graphene oxide-polysulfone filters for tap water purification, obtained by fast microwave oven treatment
}

Downloaded from: https://research.chalmers.se, 2023-04-26 10:16 UTC

Citation for the original published paper (version of record):

Kovtun, A., Zambianchi, M., Bettini, C. et al (2019). Graphene oxide-polysulfone filters for tap water purification, obtained by fast microwave oven

treatment. Nanoscale, 11(11): 22780-22787. http://dx.doi.org/10.1039/c9nr06897j

N.B. When citing this work, cite the original published paper. 


\title{
Graphene oxide-polysulfone filters for tap water purification, obtained by fast microwave oven treatment.
}

\author{
Alessandro Kovtun, ${ }^{\mathrm{a}}+$ Massimo Zambianchi, ${ }^{\mathrm{a}}+$ Cristian Bettini, $^{\mathrm{a}}$ Andrea Liscio, ${ }^{\mathrm{b}}$ Massimo Gazzano, ${ }^{\mathrm{a}}$, \\ Franco Corticelli, ${ }^{b}$ Emanuele Treossi, ${ }^{a}$ Maria Luisa Navacchia, ${ }^{a}$ Vincenzo Palermo, ${ }^{a},{ }^{*}$ Manuela Melucci \\ $\mathrm{a},{ }^{*}$
}

The availability of clean, pure water is a major challenge for the future of our society. 2-dimensional nanosheets of $\mathrm{GO}$ seem promising as nanoporous adsorbent or filters for water purification; however, their processing in macroscopic filters is challenging, and their cost vs. standard polymer filters is too high. Here we describe a novel approach to combine graphene oxide (GO) sheets with commercial polysulfone (PSPSU) granules for improved removal of organic contaminants from water. The adsorption physicphysics of contaminants on the PSPSU-GO composite follows theLangmuir and Brunauer-Emmet-Teller (BET) modelmodels, with partial swelling and intercalation of the molecules in between GO layers. Such mechanism, well-known in layered clays, has not been reported previously for graphene or GO. Our approach requires minimal amounts of GO, deposited directly on the surface of the polymer, followed by stabilization using microwaves or heat. The purification efficiency of the PSU-GO-PS composites is significantly improved vs. benchmark commercial PSPSU, as demonstrated by removal of two model contaminants, Rhodamine B and Ofloxacin. The excellent stability of the composite is confirmed by extensive ( 100 hours) filtration tests $(100 \mathrm{~h})$ in commercial water cartridges.

The outstanding adsorption efficiency of graphene oxide (GO) toward organic molecules has been widely proved in the last few years. ${ }^{1-7}$ The high surface area and the multiple surface chemical groups enable strong interface interactions by $\pi-\pi$ stacking through the $\mathrm{sp}^{2}$ region, by $\mathrm{H}$ bond through the carboxyl, hydroxyl or epoxy groups as well as van der Waals and hydrophobic interactions. . ${ }^{3,8,9}$ Such unique features have motivated the extensive use of graphene (both $\mathrm{GO}$ and reduced $\mathrm{GO}, \mathrm{rGO}$ ) and polymer-GO/rGO 3D composites such as hydrogel/aerogel, ${ }^{10,11}$ sponges, ${ }^{12-14}$ membranes ${ }^{15-20}$ as adsorbent for water remediation. The plethoralarge amount of reported data is highlightinghighlights graphene and GO as the most promising nanomaterials for the development of advanced water treatment technologies, both drinking and wastewater, technologies. ${ }^{21-23}$ Key advantages of hybrid GO based 3D structures are: i) the easy recovering of the adsorbent after use, ii) the significant enhancement of adsorption performance by addition of $\mathrm{GO}$ at even at low amount, iii) the possible modification of GO for promoting selective binding processes. ${ }^{24-30}$ 


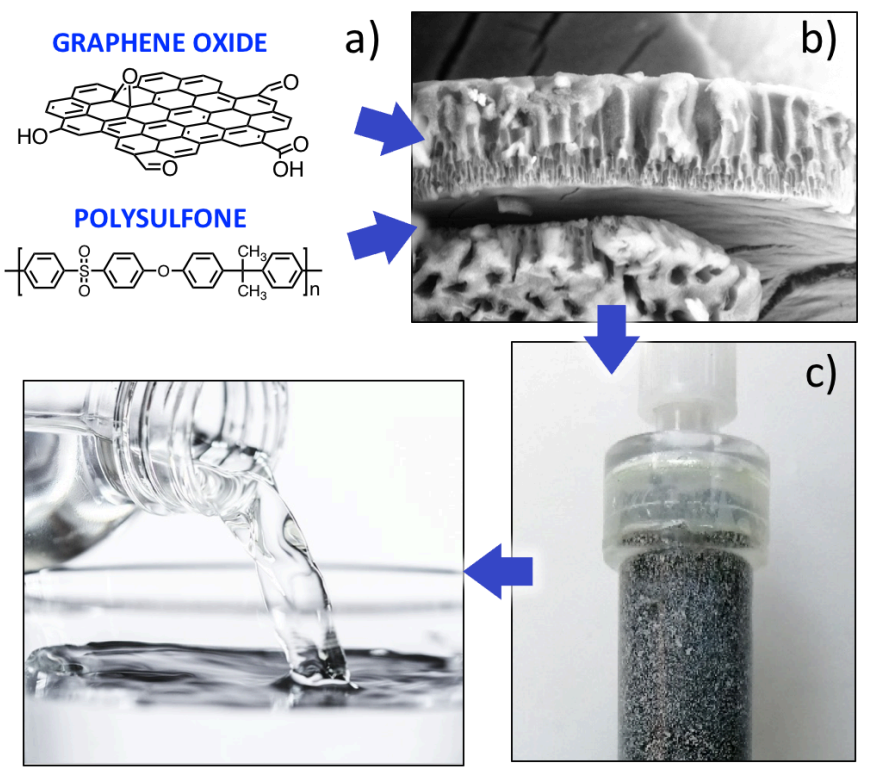

a)
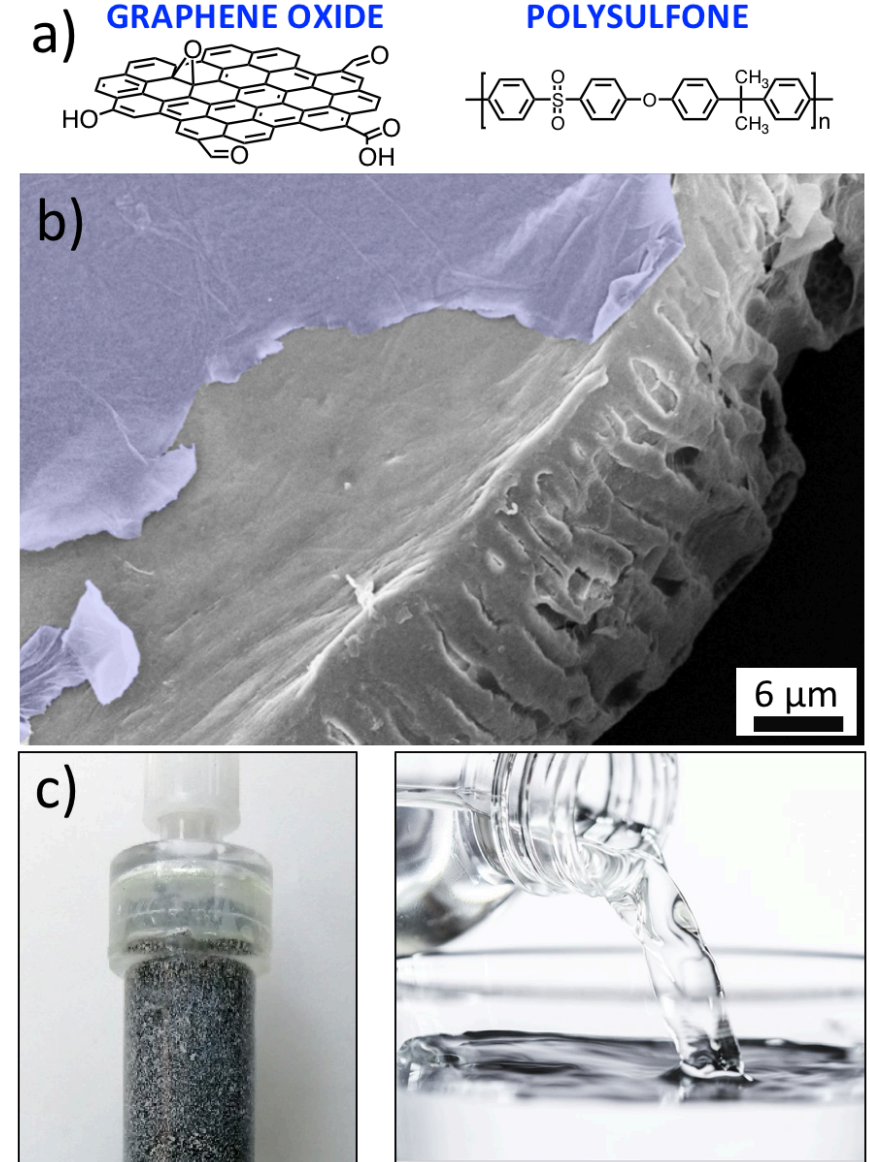

Figure 1. a) Representative chemical structure of GO and PSPSU. b) SEM and c) optical image of the PSPSU-GO composite filter. The GO sheets have been highlighted in blue in b). SEM unfiltered images are available in SI.

We recently demonstrated the superior performance of polysulfone-GO (PSPSU-GO) composites for the removal of several classes of emerging organic contaminants from tap water (figure 1). ). ${ }^{31,32}$ These compounds are currently cause of major environmental concern due to their increasing occurrence in surface water bodies and even drinking water and to their potential effect on human health and ecosystem. ${ }^{33-35}$ The composite was obtained by phase inversion of a PSPSU-GO mixture ( $5 \% \mathrm{w} / \mathrm{w}$ of GO) using water and N-methyl-2-pyrrolidinone (NMP) as orthogonal solvents, i.e. the standard industrial 
method for the preparation of PSU ultrafiltration membranes. The contaminants captured could then be removed by simple washing of the composite in ethanol, ${ }^{31}$ allowing in this way to recyclereuse the filtersmaterial.

A major challenge in developing new materials for filters for water filter remediation is to ensure their safety, i.e. utilize cheap and environment friendly methods to produce them, and stabilize the material so that no poisonous debris or molecules are released, even after extensive use.

Building on our previous results, here we describe a new method to stabilize GO nanosheets on PS using microwaves or heath. The procedure is entirely performed in water. The method is based on directly coating GO oncoat the surface of cheap industrial scraps of PS.PSU hollow fibers with $\mathrm{GO}^{32}$ Instead of using phase inversion, we fix the GO nanosheets on the polymer by use of microwaves or thermalconventional heat treatments. The procedure is entirely performed in water.

Such approach allows to produce grams scale of PSPSU-GO composite powdersmaterial with no need of toxic solvents., on PSU filters already commercialized. We then testtested the ability of such composites to capture two important organic contaminants (a textile dye and a medical drug) comparing themin comparison with standard, commercial PSunmodified PSU granules, unravellingthis allowing to unravel the physics of the adsorption process. We finally testFinally, we tested the stability of the composite upon prolonged water flow operation, by extensive test in water purification filters.

\section{Coating of GO on PSPSU recycled granules}

We used as starting substrate the scraps of anthe industrial production of polysulfone filtrationhollow fibers ultrafiltration membranes; made of PSU (representing about $10 \%$ of the total yearly production); this substrate, even if more challenging and complex than polymeric substrates we used in previous works, ${ }^{36,37}$ has the key advantage, as industrial waste, of having negative costs and represents an environmental added value of the final composite. ${ }^{32}$ Polysulfone hollow fiber (MediSulfone ${ }^{\circledR}$ ) scraps were mechanically grounded to obtain polysulfone hollow porous granules (figure S1a-c, ESI). Polysulfone hollow fiber scraps (MediSulfone) were mechanically ground to obtain polysulfone hollow porous granules (figure S1c).

The granules were then dispersed in a solution of GO in DI water; $\mathrm{GO}$ concentration was tuned to have 5 wt \% GO content in the final composite. Water was removed by heating at $50{ }^{\circ} \mathrm{C}$ on a rotary evaporator.

The granules were then dispersed in a solution of GO in DI water, prepared as described in ESI; GO concentration was tuned to have $5 \mathrm{wt} \% \mathrm{GO}$ content in the final composite in order to allow direct comparison with what already described in previous work. ${ }^{31}$ Water was removed by heating at $50{ }^{\circ} \mathrm{C}$ on a rotary evaporator.

\section{Stabilization of GO coatings on PSPSU}

A main challenge in graphene-polymer composites is to ensure a good interaction between the nanosheets and the polymer, typically to enhance mechanical properties. ${ }^{38}$ In composites for water filterfilters, a good interaction is required to ensure a long-lasting adhesion of the nano-additives to the matrix, to avoid their release in the treated water. Graphene and GO are excellent adsorbers of microwaves, and we exploited this opportunity in the past to achieve fast, environmentally free functionalization of $\mathrm{GO} .{ }^{39}$ Here, we do not use microwaves for a specific functionalization ofto functionalize GO in solution, but to ensurefix GO nanosheets to the PSU scraps, thus ensuring a good cohesion ofperformance and stabilizing the composite.

The PSstabilization was performed on the PSU-GO powder was irradiated withby microwaves at atmospheric pressure in a CEM Discover SP apparatus $(f=2.45 \mathrm{GHz})$ which has in situ magnetic variable 
speed, irradiation monitored by PC computer, infrared temperature measurement and continuous feedback temperature control. Samples were irradiated for $45 \mathrm{~min}$ at $100 \mathrm{~W}$ (fixed power).. During MW irradiation the temperature was measured, and never exceeded $70^{\circ} \mathrm{C}$. The PSPSU-GO was finally washed in a 1:1 mixture of water/ethanol to remove any traces of unreacted GO and finally left to dry at room temperature until weight stabilized. (PSU-GO-MW, Figure 1).

In an alternative approach, PSPSU-GO was also treated in a standard oven for 2 hours at $120^{\circ} \mathrm{C}$. The material was allowed to cool to room temperature, then washed and dried as described above. (figure S1d, ESI).

Remarkably, the The presence of GO coating was stable only after the MW or oven annealing treatmentconfirmed by high resolution Scanning Electron Microscope (SEM) images (figure S2)., ESI). No changes in surface porosity and cross-section were observed in comparison to pristine PS granules after coating, as measured by standard techniques ( $\mathrm{N}_{2}$ adsorption).

Remarkably, both treatments enhanced greatly the stability of GO on PSU, as shown by extended immersion in water (figure S3, ESI).

The pristine PSPSU-GO composite, the composite treated with microwaves and the one treated in oven (named respectively Blank, PSGO PSU, PSU-GO-MW and PSGOPSU-GO-OV) were then characterized by XPSXRD and XRDXPS.
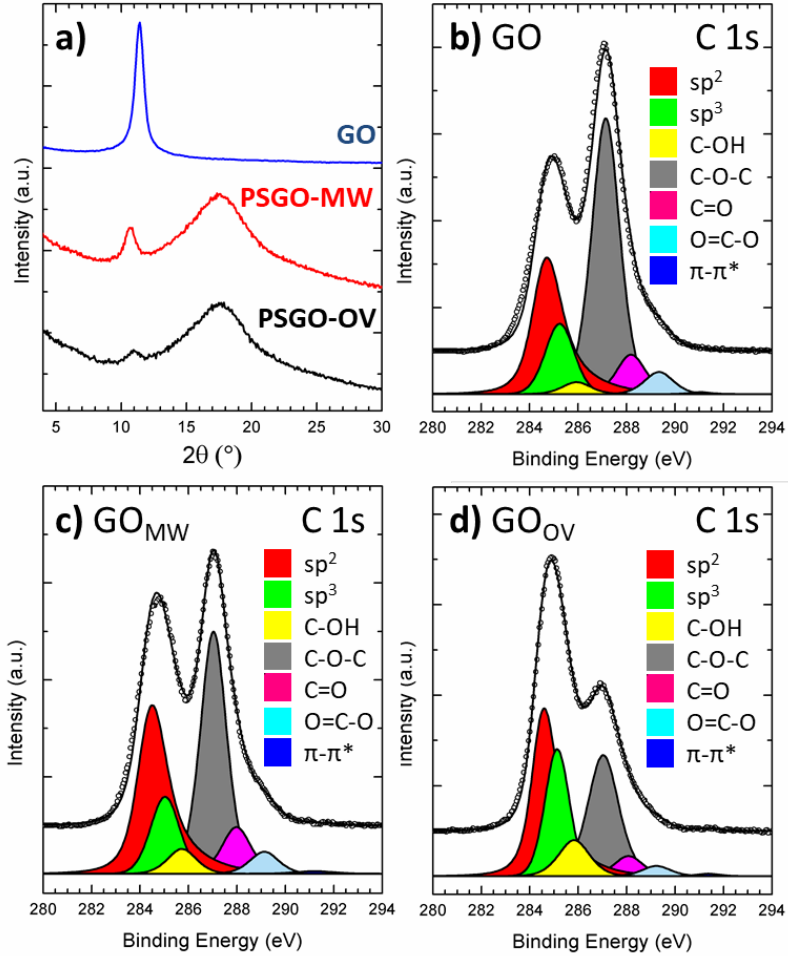
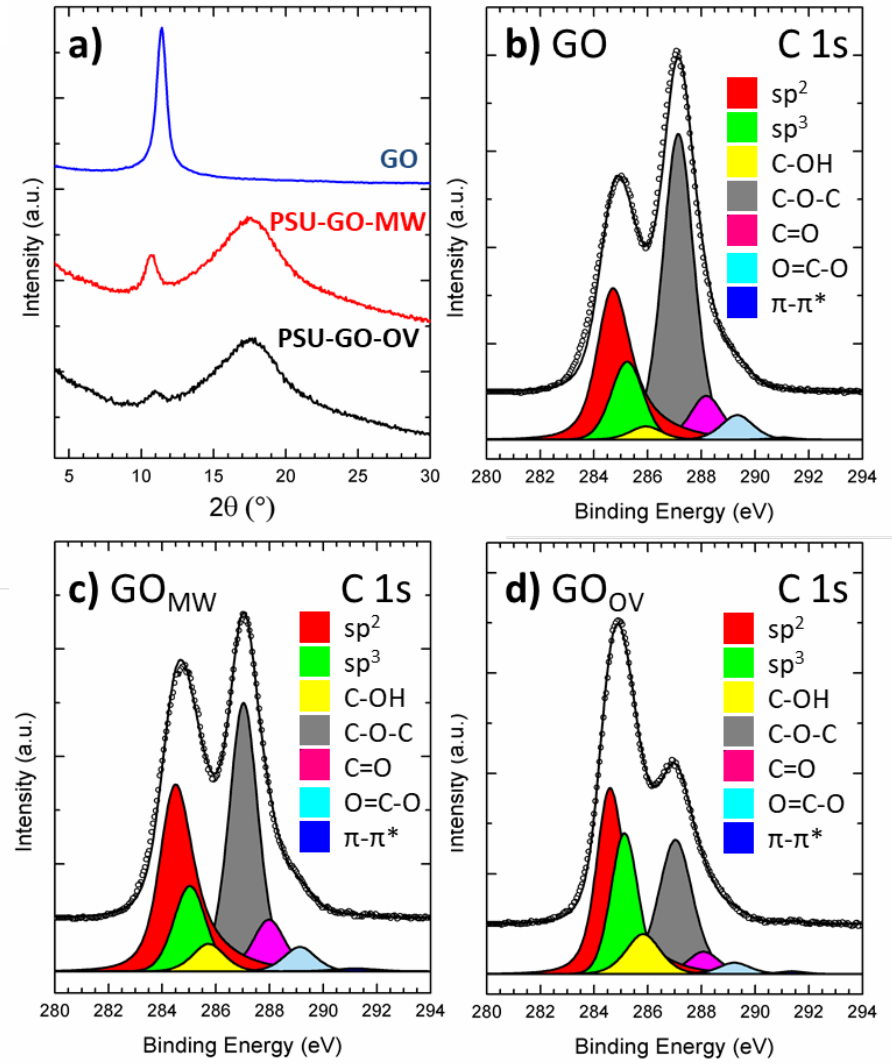

Figure 2. (a). XRD pattern of GO (blu line), PSGOPSU-GO-MW (red line) and PSGOPSU-GO-OV (black line). The $(001)$ peak of the GO structure is well appreciated in both PSGOPSU-GO samples, at 10-12', together with the amorphous bell-shaped band due to PS.PSU at 15-20'. XPS C 1s spectra of b) pristine GO, c) PSGOPSU-GO-MW samples after polymer dissolution with DCM and d) PSGOPSU-GO-OV samples after polymer dissolution with DCM. 


\section{Structural characterization of the PSGOPSU-GO composites}

$\mathrm{X}$-ray diffraction patterns were collected in Bragg-Brentano geometry (CuK radiation, $0.15418 \mathrm{~nm}$ ). Average thickness of GO crystal stacks was calculated from peak width by using the Scherrer equation. The number of $\mathrm{GO}$ layers was estimated as the ratio of the size and the interlayer distance, obtained from position of (001) GO peak.

Figure 2a compares the XRD of pure GO with the PSGOPSU-GO-MW and PSGOPSU-GO-OV composites. Pure GO showed a sharp, intense peak, as expected for a bulk 2D material. An average spacing of $7.8 \pm$ $0.3 \AA$ was observed, with average of $14 \pm 2 \mathrm{GO}$ layers stacked on top of each other.

PSGOPSU-GO-MW and PSGOPSU-GO-OV samples showed the amorphous bell-shaped band due to PSPSU, but also a clear (001) peak of the GO structure, indicating the presence of stacked GO multilayers on the surface of the PSPSU granules. The stacking distance was $8.3 \pm 0.3 \AA$ for PSGOPSU-GO-MW and $7.9 \pm 0.3 \AA$ for PSGOPSU-GO-OV; the estimated number of layers was ca. 10 layers, slightly lower than what observed in blank sample (see Table S2S1, ESI for full details and exp. errors).

The fast microwave or oven treatments did not change the mesoscale structure of the membranes, with no deformation or destruction of the PSU microchannels as observed by SEM. No changes in surface porosity and cross-section were observed in comparison to pristine PSU granules after coating, as measured by standard techniques ( $\mathrm{N}_{2}$ adsorption).

The differences between the two methods were instead observed clearly by XPS.

\section{Chemical characterization of the PSGOPSU-GO composites}

XPS is a powerful technique, able to probe the outer surface of the granules, thus allowing to get information on the possible presence of the GO coating, its uniformity, its oxygen/carbon ratio and the abundance of different chemical groups. We performed XPS on all the samples, and analysed the results with a novel deconvolution procedure which allow to obtain precise chemical analysis and oxygen content in $\mathrm{GO}$ also in presence of other oxygen-containing materials (figureFigure $2 b, c, d$ and Table 1S2). ${ }^{40}$ Such procedure is based on quantitative line-shape analysis of $C 1$ s signals with asymmetric pseudo-Voigt line-shapes (APV), in contrast to Gaussian-based approaches conventionally used (see ESI and ref. ${ }^{40}$ for details).

The XPS of untreated, bulk PSPSU granules yielded carbon/oxygen and sulphur/carbon $\mathrm{O} / \mathrm{C}=0.13 \pm 0.01$ and $\mathrm{S} / \mathrm{C}=0.04 \pm 0.01$ in good agreement with PSPSU chemical composition $(\mathrm{O} / \mathrm{C}=0.15$ and $\mathrm{S} / \mathrm{C}=0.04$, respectively).

XPS of pristine GO (Figure $2 \mathrm{~b}$ ) showed the presence of $\mathrm{sp}^{2}$ areas and several chemical groups typical of $\mathrm{GO}$, with an average oxygen/carbon ratio $\mathrm{O} / \mathrm{C}=0.39 \pm 0.01$. (see also figs. $\mathrm{S} 4,5$ and table $\mathrm{S} 2, \mathrm{ESI}$ ).

XPS technique probes only the outer surface $(2-3 \mathrm{~nm})$ of the powders; thus, from the contribution to XPS data of GO and PSPSU we could estimate the amount of surface composed of uncoated PSPSU and the one coated from the GO nanosheets. The GO coating covered $>50 \%$ of the surface of the granules; however, it was not possible to obtain a more precise estimateestimation.

To overcome this issue and have a detailed analysis of the GO coating, we thus had to remove completely the polymer phase. This was achieved by immersion in dichloromethane (DCM). This); PSU is soluble in DCM while GO is stable in such solvent completely removes, allowing a more precise characterization of the PS, while leaving unaltered the oxidation state of GO. remaining GO by XPS. For comparison sake, pure GO was also immersed in DCM and analyzedanalysed by XPS. Table 1 S2 shows the $\mathrm{O} / \mathrm{C}$ ratio measured for all chemical composition of the different samples, including their $\mathrm{O} / \mathrm{C}$ ratio. We see that, while treatment with $M W$ does not alter significantly the $G O$ chemistry $(O / C=0.38 \pm 0.01)$, 
classical heating in oven, even if at a relatively low temperature of $120^{\circ} \mathrm{C}$, gives partial reduction of the $\mathrm{GO}$, with the $\mathrm{O} / \mathrm{C}$ ratio decreasing to $0.30 \pm 0.01$.

Table 1: Oxygen/Carbon ratio measured by fitting the XPS C 1 s peak on different samples. Exp. error is \pm 0.01 in all samples.

\begin{tabular}{|l|l|}
\hline Material & $\mathrm{O} / \mathrm{C}$ ratio \\
\hline Blank PS * & 0.13 \\
\hline GO - pristine & 0.39 \\
\hline GO washed in DCM & 0.40 \\
\hline $\begin{array}{l}\text { GO } \\
\text { after }\end{array}$ \\
$\begin{array}{l}\text { DCM dissolution in } \\
\text { GO } \\
\text { after PS dissolution in } \\
\text { DCM }\end{array}$ & 0.38 \\
\hline
\end{tabular}

* O/C of blank PS was obtained from the area ratio of $\mathrm{O} 1 \mathrm{~s}$ and $\mathrm{C} 1 \mathrm{~s}$ due to the significant presence of SO groups in PS structure.

\section{Contaminants adsorption tests}

The adsorption capacities of blank PS, PSGOPSU, PSU-GO-MW and PSGOPSU-GO-OV samples were studied by exposing them to tap water contaminated with different emerging organic contaminants (EOC). The removal performance of PSU-GO towards many widely used contaminants (i.e. drugs, pigments, surfactants etc.) was previously described in ref. ${ }^{31}$; here we focused more on the preparation technique of the composite and on their application in water cartridges. As test contaminants, we chose Rhodamine $\mathrm{B}$ (RhB, a dye largely used in textile and pharmaceutic industries), and ofloxacine (OFLOX, a quinolonic antibiotic). Structure of both molecules is shown in Figure 3.

Both molecules are of significant concern for pollution of surface water bodies., ${ }^{41}$ Structure of both molecules is shown in figure 3. and adsorption capacity of OFLOX and RhB has been already reported for GO sheets. ${ }^{42,43}$

Using $100 \%$ GO in the cartridges to remove such molecules would be effective, but bulk GO in powder cannot be extruded or processed in pellets or fibers, it can be volatile and also burn or explode in suitable conditions. High adsorption capacityprice of OFLOX and RhB wasthe filtration cartridge and problems of material aggregation or mechanical stability would also hinder straightforward application of GO, making it incompatible with actual cartridge production technique, which is based on PSU fibers. Conversely, the advantage of our method is that can be applied not only on commercial membranes already reported for GO sheets. ${ }^{42,43}$ We thusproduced on large scale, but also on scraps deriving from their preparation, as demonstrated in the previous sections.

We tested the adsorption performance of pure GO, blank PS, PSGOPSU, PSU-GO-MW and PSGOPSU-GOOV composites by dedicated experiments performed in tap water contaminated on purposespiked with the target molecules(Figs. S10, ESI).. Experimental data were then fitted using Brunauer-Emmett-Teller (BET, eq. 1), Langmuir (eq. 2) and Freundlich models, see tables S5-S10. Details of HPLC method used for analytical determination are reported in ESI. 
The adsorption isotherm of GO was performed at fixed concentration of RhB and OFLOX by varying the amount of GO. The two contaminants (in powder, as received) were added to $\mathrm{GO}$ suspensions ( $5 \mathrm{ml}$ ) at different concentrations. The details of sample preparation and a list of samples prepared are reported in tables S3 and S4. The isotherm curves were obtained equilibrating the solutions for $24 \mathrm{hrs}$ at room temperature. The solutions were analysed by HPLC.

The adsorption isotherms of blank PS, PSGO-MW and PSGO-OV were fitted with the Brunauer-EmmettTeller (BET) adsorption model (eq. 1, figure 3). The BET model describes a multilayer adsorption mechanism in the gas-solid and liquid-solid equilibrium systems. described by the formula: ${ }^{44}$

$Q_{e}=\left(Q_{m} \cdot C_{B E T} \cdot x\right) /\left((1-x) \cdot\left(1+C_{B E T} \cdot x-x\right)\right)$

Here $Q_{e}(\mathrm{mg} / \mathrm{g})$ is the quantityamount of molecules adsorbed when the equilibrium concentration is $C_{e}$ $(\mathrm{mg} / \mathrm{mL}) ; \mathrm{Q}_{\mathrm{m}}$ is the, the quantity amount of molecules needed to cover the entire adsorbent surface with a monolayer (i.e. the monolayer saturation capacity, $\mathrm{mg} / \mathrm{g}$ ). The thermodynamic equilibrium BET constant is $C_{B E T}=\exp \left(\Delta E / K_{b} T\right)$, where $\Delta \mathrm{E}$ is the difference between the energy of adsorption of first and second layers of molecules, $K_{b} T$ is the termalthermal energy; $\mathrm{x}=\mathrm{Ce} / \mathrm{Cs}$, where $\mathrm{Cs}(\mathrm{mg} / \mathrm{mL})$ is the adsorbate saturation concentration in BET adsorption process (see ref. ${ }^{45}$ ). The linear fit was performed plotting $y=1 /\left(Q_{e} \cdot(1 / x-1)\right)$ vs. $x$ and the results are shown in figure 3 and in table $S 3 x$.

Langmuir model describes a monolayer adsorption process, which depends on the equilibrium concentration $C_{e}(\mathrm{mg} / \mathrm{mL})$; the $Q_{m}(\mathrm{mg} / \mathrm{g})$ and the thermodynamic equilibrium constant $\mathrm{K}_{\mathrm{L}}(\mathrm{mL} / \mathrm{mg})$.

$Q_{e}=Q_{m} \cdot K_{L} \cdot C_{e} /\left(1+K_{L} \cdot C_{e}\right)$

Remarkably, Eq. 1 can fit very well all adsorption isotherms tested withof Ofloxacine on the 3three different materials and 2 target molecules.. This indicates that the adsorption follows in all these cases the BET physical model, i.e. can form multilayers on the substrate, filling small pores first.

Once assessing thatOn the same physicalother hand, the adsorption of RhB on the 3 different materials can be better described by Langmuir model applies(eq. 2). It should be noted anyhow that both molecules show a good fit to both BET and Langmuir models (tables S8-S10, ESI). Freundlich isotherm model gave instead a poor fit for all the surfaces under test, we compared their performance in capturing RhB and OFLOX in solution. samples.

Figure 3 shows that both PSGOPSU-GO composites outperform the standard PS polymerPSU material. In particular, PSGOPSU-GO-MW samples captured ca. 3 times more RhB than blank, and >ca. 2 times more OFLOX than PSU.

We also comparedlt is interesting to compare the adsorption of RhB and OFLOX in solution with adsorption of $\mathrm{N}_{2}$ molecules from gas, which is the conventional technique used to measure the specific surface area (SSA) of nanomaterials (table S11, ESI. 
a) RHODAMINE B

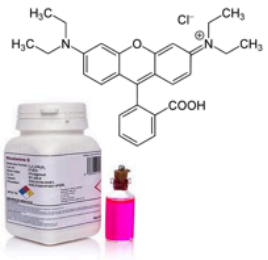

b) OFLOXACIN

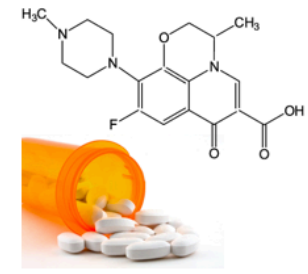

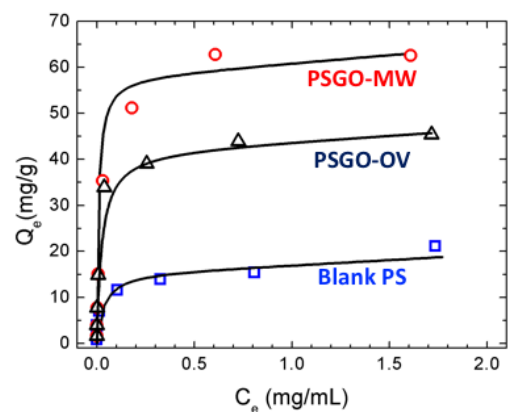

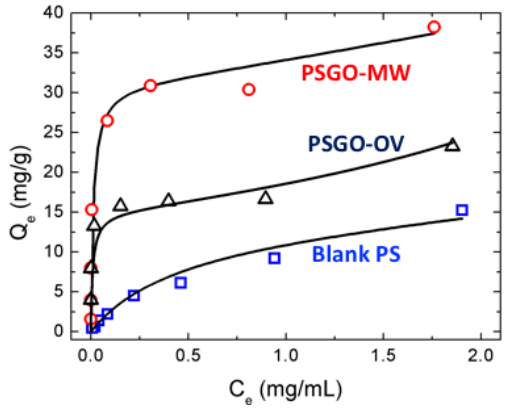

a) RHODAMINE B

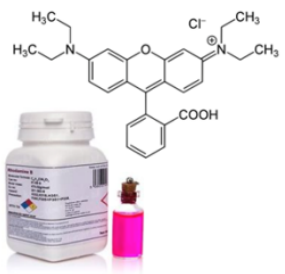

b) OFLOXACIN

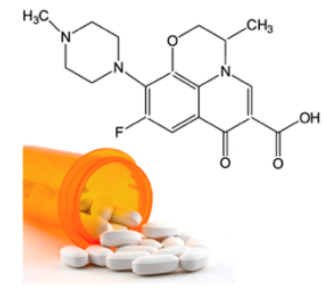

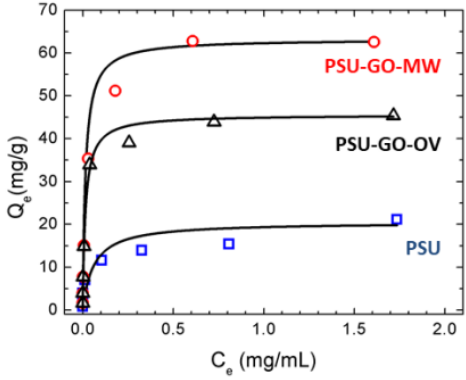

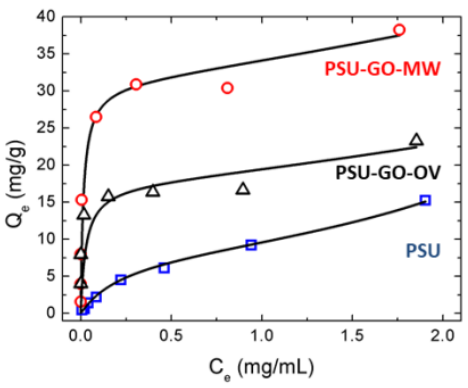

Figure 3. Chemical formulasstructures of RhB (a) and OFLOX (b), and the corresponding adsorption isotherms. Black lines represent Langmuir model for RhB (a) and BET model for OFLOX (b).

The two processes are very different; $N_{2}$ adsorbs from gas phase, with weak interaction between $\mathrm{N}_{2}$ molecules and the substrate, mainly due to Vanvan der Waals interactions. In solution, instead, molecules adsorb by displacing other solvent molecules already present on the surface, ${ }^{46}$ often interacting strongly with the substrate and the solvent due to electrostatic forces.

That'sThis explains why the surface area measured by gas adsorption of $\mathrm{GO}^{47}$ and more generally of layered materials, ${ }^{48}$ is systematically smaller than what measured by adsorption of molecules in solution. To compare the adsorption capability of different molecules, we converted the quantity of molecules adsorbed $Q_{m}$ (measured above in $\mathrm{mg} / \mathrm{g}$ ) in the SSA ideally occupied by those molecules using:

$S S A=Q_{m} M_{w}^{-1} N_{A} A_{m o l}$

where $M_{w}$ is the molecular weight, $N_{A}$ is the Avogadro number and $A_{m o l}$ is the estimated "footprint" of the molecule, i.e. the amount of substrate ideally occupied by a single adsorbed molecule. We could estimate $A_{\text {mo }} \approx 1.81 \mathrm{~nm}^{2}$ for RhB and $A_{m o} \approx 1.30 \mathrm{~nm}^{2}$ for OFLOX, based on STM ${ }^{49}$ orand XRD measurements ${ }^{50}$ respectively. This is just an approximation, and the actual footprint of the molecules on the surface will likely vary due to specific interactions between the molecule, the solvent and $\mathrm{GO}^{51}$ We expect thoughtthough that the footprint will remain of the same order of magnitude of what was previously reported. ${ }^{49} 50$

Figure 4a compares the estimated SSA of blank PS, PSGOPSU, PSU-GO-MW and PSGOPSU-GO-OV, estimated by molecular adsorption of $\mathrm{N}_{2}$ from gas, RhB and OFLOX from water solutions. We underline that the SSA reported should be considered as specific to the material studied, to be used only for direct comparison among samples, because it depends not only on the material, but also on its interaction with the molecule considered. 
a) Area available to molecular adsorption

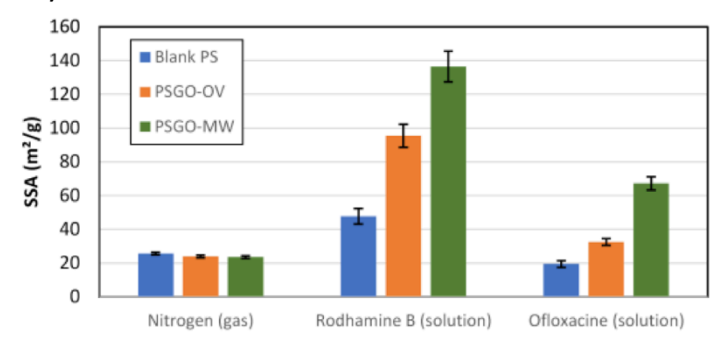

Area available to molecular adsorption

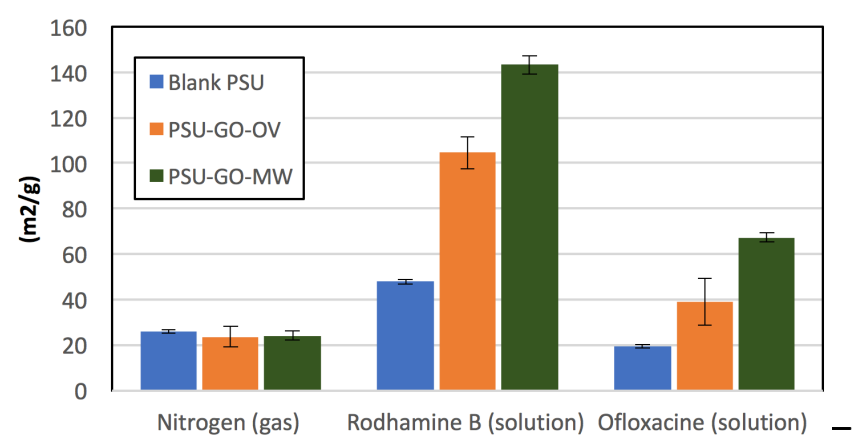

b)

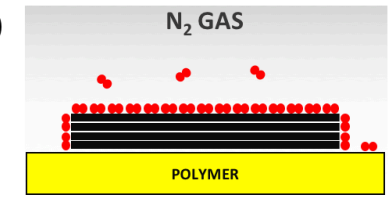

c)

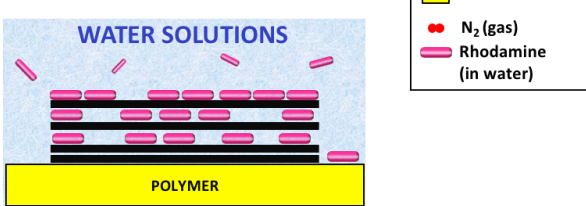

Figure 4. a) Comparison of the area measured in each specific molecule/substrate combination tested. Conversion from $\mathrm{mg} / \mathrm{g}$ to $\mathrm{m}^{2} / \mathrm{g}$ has been performed estimating molecular footprint, as described in main text. b,c) Schematic cartoon showing the different possible capture mechanism of $N_{2}$ in gas and of organic molecules in solutions (see also ref. ${ }^{48}$ )

In case of nitrogen adsorption from gas, all three materials give comparable SSA values, $\approx 23-2526 \mathrm{~m}^{2} / \mathrm{g}$, confirming that the GO coating and fixation does not change the overall porosity of the material, and that $\mathrm{N}_{2}$ adsorption does not depend significantly on the surface chemistry of the different samples. OFLOX adsorption on pristine, blank PSPSU gives SSA $=20 \pm 2 \mathrm{~m}^{2} / \mathrm{g}$, a value comparable to what measured with nitrogen; conversely, upon GO coating the number of molecules which can be captured by the material increases significantly. The estimated SSA increases to $3339 \pm 2 \mathrm{~m}^{2} / \mathrm{g}$ for the oventreated PSU-GO, and to $67 \pm 4 \mathrm{~m}^{2} / \mathrm{g}$ for the MW treated PSU-GO, ca. three times the original value. RhB gives even more extreme changes, likely due to its electric charges (being a salt).. The original SSA measured on blank (48 $\pm 5 \mathrm{~m}^{2} / \mathrm{g}$ ) increases to $95105 \pm 7 \mathrm{~m}^{2} / \mathrm{g}$ for oven treated PSU-GO, and to $135143 \pm$ $9 \mathrm{~m}^{2} / \mathrm{g}$ for the microwave treated sample, corresponding to an adsorption capacity of $6063 \mathrm{mg}$ of Rhodamine per gram of samplecomposite. Such SSA is more than five times larger than the SSA measured with nitrogen $\left(24 \mathrm{~m}^{2} / \mathrm{g}\right)$. Such large difference cannot be explained uniquely by the uncertainty in the detailed molecular arrangement of the molecule on the substrates; furthermore, both RhB and OFLOX give significantly different SSA on different substrates, while adsorption of $\mathrm{N}_{2}$ from gas gives similar values for PSGO, PSGO-MW and PSGO-OV.all samples. 
Similarly, tests performed on $100 \%$ GO, with no PS, gave extremely high values of SSA, reported in table $\mathrm{S} 6$ (though if the use of pure GO would not be economically viable for filters).

The differences observed in figure 4 suggest that $\mathrm{GO}$ can act as an effective adsorbent for organic molecules thanks to its layered structure, able to capture the target pollutants for more than $6 \%$ of its weight in the best scenario (RhB on microwave treated samples). Adsorption test performed on pure GO also gave very high values of SSA (table S5).

It is known from characterization of layered minerals like clay that the presence of swelling minerals should be suspected when the SSA measurements in liquid are significantly higher than gas adsorption measurements. ${ }^{48} \mathrm{GO}$ is a unique layered material, which can easily be exfoliated in water; ${ }^{52}$ water molecules can intercalate and travel efficiently among stacked GO nanosheets. ${ }^{53}$ Water trapped in between the GO nanosheets can behave as bulk water. ${ }^{54}$

To demonstrate this hypothesis, we performed XRD scans on PSGOPSU-GO-OV composites exposed to a large excess of RhB contaminants (figure S9 inS8, ESI). In the exposed samples the (001) peak of GO almost disappeared, a clear indication that the organic molecules were disrupting the periodic stacking of the nanosheets. While intercalation of ions or molecules can give XRD peaks in graphite intercalation compounds, no periodicity could be observed in the PSGOPSU-GO samples exposed to RhB, likely due to the small number of nanosheets involved and the uneven intercalation of RhB within the GO layers.

\section{GO stability, measured by release tests}

Any material used for water purification should be safe, i.e. should not release additional contaminants in the outgoing water. A complete study of adsorbent materials should not only measure how much contaminants can transfer from the water to the filter; it should also demonstrate that there is no transfer of contaminants from the filter to the water. We observed in target experiments (figure S6S10, ESI) that our GO is "intrinsically safe",, while being highly soluble in distilled water, butis insoluble in tap water, thus being "intrinsically safe" for this specific application.

In addition to this evidence, we decided to test any possible release of GO by inserting the composite samples in a commercial water-filter cartridge $\mathrm{e}^{55}$ and flowingcirculating water (both $\mathrm{mQ}$ and tap water) through the cartridge at $2 \mathrm{~L} / \mathrm{h}$ for 100 hours.

a)
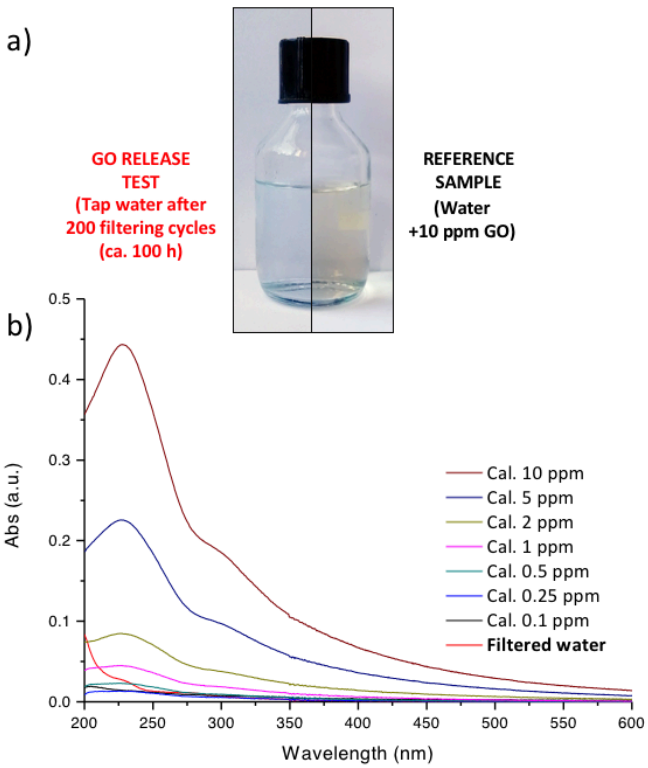

Figure 5. a) Photo of $m Q$ water filtered for $100 \mathrm{~h}$ through the PSU-GO filter compared to standard solution of GO in $\mathrm{mQ}$ water at different concentration. b) UV-Vis absorption spectra of water filtered for $100 \mathrm{~h}$ through the PSU-GO filter, compared to calibration samples of water contaminated with different amounts of GO. 
The filtered water was then analyzed to detect possible GO traces beyond such limit. For better safety assessment, we used two parallel GO detection techniques: UV-vis absorption and dynamic light scattering. We compared the filtered water to standard samples consisting of $\mathrm{mQ}$ water contaminated with known amounts of GO.

We performed UV-vis spectroscopy on water re-circulated for 100 hours through filters containing PSUGO-MW and PSU-GO-OV powders (Figure S11, ESI). Detection limit, estimated with calibrated GO solutions, was about 1 ppm.

Figure 5 shows the images of the recirculated water, as well as the UV-vis spectra of the filtered water (red line) and of calibration solutions having a concentration range 0.25-10 ppm. The comparison indicates that any GO possibly released in filtered water was below $1 \mathrm{ppm}$.

Recent work indicate that safe limits of GO concentration to avoid toxic effects are between few tens and few hundreds of $10-1000 \mathrm{ppm}$ (for a complete review on this important topic see ref. ${ }^{56}$. GO limits for aquatic organisms are in the range $40-2000$ ppm. $^{57}$

Experiments in tap water were also performed showing transparent solution even after concentration of the sample (Figure S15, ESI).

a)
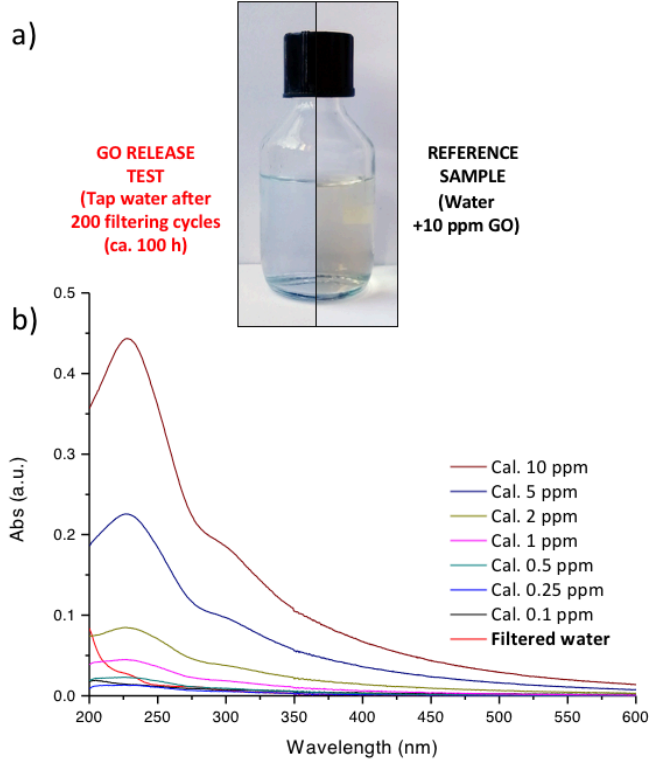

Figure 5. a) Photo of tap water filtered for $100 \mathrm{~h}$ through the PSGO filter compared to water contaminated with $10 \mathrm{ppm}$ of GO. b) UV-Vis absorption spectra of water filtered for $100 \mathrm{~h}$ through the PSGO filter, compared to calibration samples of water contaminated with different amounts of GO.

The filtered water was then analyzed to detect possible GO traces beyond such limits. For better safety assessment, we used two parallel GO detection techniques: UV-vis absorption and dynamic light scattering. We compared the filtered water to standard samples consisting of $\mathrm{mQ}$ water contaminated with known amounts of GO.

We performed UV-vis spectroscopy on water re-circulated for 50 hours through filters containing PSGOMW and PSGO-OV powders. Detection limit, estimated with calibrated GO solutions, was 0,5-1 ppm. Figure 5 shows the images of the recirculated water, as well as the UV-vis spectra of the filtered water (red line) and of contaminated calibration solutions having a concentration range 0,25-10 ppm. The comparison indicates that any GO possibly released in filtered water was below $1 \mathrm{ppm}$.

Dynamic Light Scattering (DLS) measures the auto-correlation function of photons scattered by nanoscopic objects in solution, and we used it to detect the possible presence of small particles or nanosheets released in water (see details in ESI, Figs. S4-5S13-14). Its application to non-spherical 
objects is not straightforward, but it has been successfully used for the characterization of 2dimensional materials such as graphene and BN. ${ }^{58-60}$

DLS detection limit for GO nanosheets was 5 ppm, as determined with calibration solutions of GO with known concentration (seesection 13, ESI for the DLS spectrograms and measurement details).). DLS measurements performed on tap water re-circulated for 100 hours through filters containing PSGOPSUGO-MW and PSGOPSU-GO-OV powders did not show any presence of contaminants in the size range 1 $\mathrm{nm}-10$ im for concentrations $>5 \mathrm{ppm}$, confirming that no significant amounts of GO nanosheets were released by the composite. within the limit of detection range. For comparison, blank PSPSU-GO composites with no MW or OVstabilization treatment would instead release large amounts of debris and contaminants when suspended in water, visible by naked eye (Figure S2 inS3, ESI). This demonstratedemonstrates the efficacy of the microwave processing to stabilize, at low temperature, the composite material, while keeping its adsorption performance high.

Attempts to concentrate the sample to further enhance the detection of contaminants, both by ultracentrifugation and vacuum evaporation failed (Figures S16-S17, ESI).

\section{Conclusions}

The previous sectionsdata described above demonstrate that the proposed approach is effectivelya stable and cost-effective PSU-GO composite could be obtained by fixing a thin layerlayers (ca. 10 sheets) of GO on the PSPSU granules. The process is easily up-scalable, relying only on water processing and microwaves or conventional heating activation of already produced membranes, without the need of adapting already existing production technologies and plants. In particular, microwave treatments are already extensively used on large scale in industry. The stabilization effect of the treatment appears very effective, yielding noa release of GO below detection limit upon extensive testing ( $2 \mathrm{~L} / \mathrm{h}$ for $100 \mathrm{~h}$ ) as demonstrated by two different independent techniques.

The synergic effect of GO and PSU can be demonstrated by comparing the adsorption capability of the PSU-GO composite vs. the performance of bulk GO (see also table S5 and S11 in ESI). The final effective surface area measured for Rhodamine removal by PSU-GO-MW is $143 \mathrm{~m} 2 / \mathrm{g}$, more than $10 \%$ of the area measured in pure $\mathrm{GO}$, even if only $5 \%$ of $\mathrm{GO}$ is present in the composite. A similar estimate can be done for OFL, where the performance is ca. $9 \%$ of bulk GO, for the $5 \%$ loading. The excellent performance obtained in removing the target contaminants from tap water can be attributed to the unique properties of 2D materials. Both GO and PSU can capture water organic contaminants; however, their meso-structure and capture mechanism is different. While PSU features a 3D network of meso- and micro-channels, GO features a stacked structure with nanometric spacing, which changes upon molecule intercalation (see XRD data in ESI). To work at best, such GO layered structure should be fully accessible from solution, i.e. arranged as thin layers, avoiding strong aggregation or bulk clusters as could happen in bulk graphite oxide powders. The structure described here, made of thin GO 2D coatings on a mechanically stable 3D structure of PSU microchannels (figures 1 and S2) is thus ideal to ensure that all GO can actively contribute to the contaminant removal.

Remarkably, the GO coating does not modify significantly the porous structure of the PSPSU. The standard measurements of surface area, based on weak physisorption of $\mathrm{N}_{2}$ molecules in gas, gives a similar SSA before and after the coating. However, the interaction of the composite with organic dyesmolecules in solution gives a much higher effective area of interaction, which varies with surface chemistry and target molecule structure. Quantitative analysis indicates that the adsorption process follows a BET and Langmuir model, and that the reason of improved performance is due to the fewlayers structure of the GO nanosheet, which gives a higher effective area available for adsorption vs standard bulk polymer, due to intercalation of the contaminants in between the sheets. Such process is 
not observed in standard surface area measurement using adsorption of nitrogen gas and is similar to what was previously observed in layered minerals like clays. Respect to other layered materials, the GO nanosheets have better processability, being soluble in water, and a versatile, tunable surface chemistry which can attract contaminants with $\pi-\pi$, Vanvan der Waals or hydrogen bonding interactions.

Such materialWhile microwaves have been used extensively to exfoliate or functionalize graphene, their use to "fix" GO on a polymer substrate has not been reported before (patent submitted).

Such method can be applied on micro and ultrafiltration membranes already commercially available, as well as scraps deriving from their preparation, without the need of adapting industrial production systems.

The PSU-GO composite exhibits thus an original adsorption mechanism, interesting for fundamental science, as well as promising potential application in commercial filters for water purification, a topic of timely importance. Further work is ongoing to better evaluate the industrial feasibility of PS-GO based filters (patent submitted).

\section{Acknowledgements}

The research leading to these results has received funding from the European Union's Horizon 2020 research and innovation programme under GrapheneCore2 785219 - Graphene Flagship and the Swedish Research Council (project Janus 2017-04456).

\section{Conflicts of interest}

The authors declare no conflicts of interest.

\section{References}

1. Y. Zhou, O. G. Apul and T. Karanfil, Water Res., 2015, 79, 57-67.

2. Y. Shen, Q. L. Fang and B. L. Chen, Environmental Science \& Technology, 2015, 49, 67-84.

3. G. Ersan, O. G. Apul, F. Perreault and T. Karanfil, Water Res., 2017, 126, 385-398.

4. O. G. Apul, Q. L. Wang, Y. Zhou and T. Karanfil, Water Res., 2013, 47, 1648-1654.

5. Z. Q. Niu, L. L. Liu, L. Zhang and X. D. Chen, Small, 2014, 10, 3434-3441.

6. F. Perreault, A. F. de Faria and M. Elimelech, Chem. Soc. Rev., 2015, 44, 5861-5896.

7. B. Beless, H. S. Rifai and D. F. Rodrigues, Environmental Science \& Technology, 2014, 48, 1037210379.

8. L. H. Jiang, Y. G. Liu, S. B. Liu, G. M. Zeng, X. J. Hu, X. Hu, Z. Guo, X. F. Tan, L. L. Wang and Z. B. Wu, Environmental Science \& Technology, 2017, 51, 6352-6359.

9. F. F. Liu, J. Zhao, S. G. Wang, P. Du and B. S. Xing, Environmental Science \& Technology, 2014, 48, 13197-13206.

10. F. Wang, Y. Wang, W. W. Zhan, S. R. Yu, W. H. Zhong, G. Sui and X. P. Yang, Chem. Eng. J., 2017, 320, 539-548.

11. L. Gan, H. Li, L. W. Chen, L. J. Xu, J. Liu, A. B. Geng, C. T. Mei and S. M. Shang, Colloid. Polym. Sci., 2018, 296, 607-615.

12. H. C. Bi, X. Xie, K. B. Yin, Y. L. Zhou, S. Wan, L. B. He, F. Xu, F. Banhart, L. T. Sun and R. S. Ruoff, Adv. Funct. Mater., 2012, 22, 4421-4425.

13. Y. Q. Chen, L. B. Chen, H. Bai and L. Li, Journal of Materials Chemistry A, 2014, 2, 13744-13744.

14. J. P. Zhao, W. C. Ren and H. M. Cheng, J. Mater. Chem., 2012, 22, 20197-20202.

15. R. Rezaee, S. Nasseri, A. H. Mahvi, R. Nabizadeh, S. A. Mousavi, A. Rashidi, A. Jafari and S. Nazmara, Journal of Environmental Health Science and Engineering, 2015, 13. 
16. H. Q. Wu, B. B. Tang and P. Y. Wu, Journal of Membrane Science, 2014, 451, 94-102.

17. J. A. Prince, S. Bhuvana, V. Anbharasi, N. Ayyanar, K. V. K. Boodhoo and G. Singh, Water Res., 2016, 103, 311-318.

18. M. Miculescu, V. K. Thakur, F. Miculescu and S. I. Voicu, Polym. Adv. Technol., 2016, 27, 844-859.

19. X. Y. Zhu, K. J. Yang and B. L. Chen, Environmental Science-Nano, 2017, 4, 2267-2285.

20. J. Alam, A. K. Shukla, M. Alhoshan, L. A. Dass, M. R. Muthumareeswaran, A. Khan and F. A. A. Ali, Adv. Polym. Tech., 2018, 37, 2597-2608.

21. S. Bolisetty, M. Peydayesh and R. Mezzenga, Chem. Soc. Rev., 2019, 48, 463-487.

22. C. Santhosh, V. Velmurugan, G. Jacob, S. K. Jeong, A. N. Grace and A. Bhatnagar, Chem. Eng. J., 2016, 306, 1116-1137.

23. P. Westerhoff, P. Alvarez, Q. L. Li, J. Gardea-Torresdey and J. Zimmerman, Environmental ScienceNano, 2016, 3, 1241-1253.

24. M. P. Wei, H. Chai, Y. L. Cao and D. Z. Jia, J. Colloid Interface Sci., 2018, 524, 297-305.

25. N. Oger, Y. T. F. Lin, C. Labrugere, E. Le Grognec, F. Rataboul and F. X. Felpin, Carbon, 2016, 96, 342-350.

26. J. Abraham, K. S. Vasu, C. D. Williams, K. Gopinadhan, Y. Su, C. T. Cherian, J. Dix, E. Prestat, S. J. Haigh, I. V. Grigorieva, P. Carbone, A. K. Geim and R. R. Nair, Nature Nanotechnology, 2017, 12, 546-+. 27. C. J. Madadrang, H. Y. Kim, G. H. Gao, N. Wang, J. Zhu, H. Feng, M. Gorring, M. L. Kasner and S. F. Hou, Acs Applied Materials \& Interfaces, 2012, 4, 1186-1193.

28. W. Gao, M. Majumder, L. B. Alemany, T. N. Narayanan, M. A. Ibarra, B. K. Pradhan and P. M. Ajayan, Acs Applied Materials \& Interfaces, 2011, 3, 1821-1826.

29. Y. Shen and B. L. Chen, Environmental Science \& Technology, 2015, 49, 7364-7372.

30. W. K. Chee, H. N. Lim, N. M. Huang and I. Harrison, Rsc Advances, 2015, 5, 68014-68051.

31. M. Zambianchi-nchi, M. Durso, A. Liscio, E. Treossi, C. Bettini, M. L. Capobianco, A. Aluigi, A. Kovtun, G. Ruani, F. Corticelli, M. Brucale, V. Palermo, M. L. Navacchia and M. Melucci, Chem. Eng. J., 2017, 326, 130-140.

32. M. Zambianchi, A. Aluigi, M. L. Capobianco, F. Corticelli, I. Elmi, S. Zampolli, F. Stante, L. Bocchi, F. Belosi, M. L. Navacchia and M. Melucci, Advanced Sustainable Systems, 2017, 1, \#1700019.

33. T. Laaninen, European Parliamentary Research Service, 2018, PE 625.179.

34. V. Geissen, H. Mol, E. Klumpp, G. Umlauf, M. Nadal, M. van der Ploeg, S. van de Zee and C. J. Ritsema, International Soil and Water Conservation Research, 2015, 3, 57-65.

35. S. Schulze, D. Zahn, R. Montes, R. Rodil, J. B. Quintana, T. P. Knepper, T. Reemtsma and U. Berger, Water Res., 2019, 153, 80-90.

36. D. Pierleoni, Z. Y. Xia, M. Christian, S. Ligi, M. Minelli, V. Morandi, F. Doghieri and V. Palermo, Carbon, 2016, 96, 503-512.

37. D. Pierleoni, M. Minelli, S. Ligi, M. Christian, S. Funke, N. Reineking, V. Morandi, F. Doghieri and V. Palermo, ACS Applied Materials \& Interfaces, 2018, 10, 11242-11250.

38. V. Palermo, I. A. Kinloch, S. Ligi and N. M. Pugno, Adv. Mater., 2016, 28, 6232-6238.

39. M. Melucci, E. Treossi, L. Ortolani, G. Giambastiani, V. Morandi, P. Klar, C. Casiraghi, P. Samori and V. Palermo, J. Mater. Chem., 2010, 20, 9052-9060.

40. A. Kovtun, D. Jones, S. Dell'Elce, E. Treossi, A. Liscio and V. Palermo, Carbon, 2019, 143, 268-275.

41. A. J. Ebele, M. Abou-Elwafa Abdallah and S. Harrad, Emerging Contaminants, 2017, 3, 1-16.

42. K. X. Sun, S. N. Dong, Y. Y. Sun, B. Gao, W. C. Du, H. X. Xu and J. C. Wu, J. Hazard. Mater., 2018, 348, 92-99.

43. H. Y. Guo, T. F. Jiao, Q. R. Zhang, W. F. Guo, Q. M. Peng and X. H. Yan, Nanoscale Research Letters, 2015, 10. 
44. K. S. W. Sing, D. H. Everett, R. A. W. Haul, L. Moscou, R. A. Pierotti, J. Rouquerol and T. Siemieniewska, Pure Appl. Chem., 1985, 57, 603-619.

45. A. Ebadi, J. S. S. Mohammadzadeh and A. Khudiev, Adsorption-Journal of the International Adsorption Society, 2009, 15, 65-73.

46. A. Schlierf, H. F. Yang, E. Gebremedhn, E. Treossi, L. Ortolani, L. P. Chen, A. Minoia, V. Morandi, P. Samori, C. Casiraghi, D. Beljonne and V. Palermo, Nanoscale, 2013, 5, 4205-4216.

47. M. J. McAllister, J. L. Li, D. H. Adamson, H. C. Schniepp, A. A. Abdala, J. Liu, M. Herrera-Alonso, D. L. Milius, R. Car, R. K. Prud'homme and I. A. Aksay, Chem. Mater., 2007, 19, 4396-4404.

48. J. C. Santamarina, K. A. Klein, Y. H. Wang and E. Prencke, Canadian Geotechnical Journal, 2002, 39, 233-241.

49. G.-J. Su, S.-X. Yin, L.-J. Wan, J.-C. Zhao and C.-L. Bai, Surf. Sci., 2004, 551, 204-212.

50. J. T. J. Freitas, C. C. de Melo, O. Viana, F. F. Ferreira and A. C. Doriguetto, Crystal Growth \& Design, 2018, 18, 3558-3568.

51. V. Palermo and P. Samori, Angew. Chem. Int. Ed., 2007, 46, 4428-4432.

52. E. Treossi, M. Melucci, A. Liscio, M. Gazzano, P. Samorì and V. Palermo, J. Am. Chem. Soc., 2009, 131, 15576-15577.

53. R. R. Nair, H. A. Wu, P. N. Jayaram, I. V. Grigorieva and A. K. Geim, Science, 2012, 335, 442-444.

54. G. Romanelli, A. Liscio, R. Senesi, R. Zamboni, E. Treossi, F. Liscio, G. Giambastiani, V. Palermo, F. Fernandez-Alonso and C. Andreani, Carbon, 2016, 108, 199-203.

55. Medica, https://www. medica.it/.

56. A. Bianco, Angew. Chem. Int. Ed., 2013, 52, 4986-4997.

57. J. Zhao, Z. Y. Wang, J. C. White and B. S. Xing, Environmental Science \& Technology, 2014, 48, 9995-10009.

58. K. Kouroupis-Agalou, A. Liscio, E. Treossi, L. Ortolani, V. Morandi, N. M. Pugno and V. Palermo, Nanoscale, 2014, 6, 5926-5933.

59. A. Catheline, L. Ortolani, V. Morandi, M. Melle-Franco, C. Drummond, C. Zakri and A. Penicaud, Soft Matter, 2012, 8, 7882-7887.

60. M. Lotya, A. Rakovich, J. F. Donegan and J. N. Coleman, Nanotechnology, 2013, 24, \#265703. 